\title{
Subependymal hemorrhage following drainage of chronic subdural hematoma: Probable causative mechanisms and prevention strategies
}

\author{
Amey R. Savardekar, Pravin Salunke \\ Department of Neurosurgery, Post Graduate Institute of Medical Education and Research, Chandigarh, Punjab, India
}

\section{ABSTRACT}

Chronic subdural hematoma (cSDH) is a common clinical entity treated by neurosurgeons with good cure rates. Recurrence of the hematoma and failure of the brain to re-expand are commonly observed complications following surgery for cSDH. An intracerebral or intraventricular hemorrhage following evacuation of cSDH is very rarely observed, albeit with devastating consequences. We report the finding of a subependymal hemorrhage after evacuation of a cSDH and discuss the probable causative mechanisms underlying this rare occurrence and the prevention strategies to avoid it.

Key words: Chronic subdural hematoma, intracerebral hematoma, post-operative intracerebral hematoma

\section{Introduction}

Chronic subdural hematoma (cSDH) is a common clinical entity treated by neurosurgeons with very good cure rates. ${ }^{[1]}$ Recurrence of the hematoma, infection, seizure, cerebral edema, tension pneumocephalus and failure of the brain to re-expand are well-recognized complications following surgery for $\mathrm{cSDH} .{ }^{[2]}$ An intracerebral or intraventricular hemorrhage following such an evacuation is very rarely observed (incidence $\sim 0.7-5 \%$ ), albeit with devastating consequences. ${ }^{[2-4]}$ We report the finding of a subependymal hemorrhage after evacuation of a cSDH and discuss the probable causative mechanisms underlying this rare occurrence and the prevention strategies to avoid it.

\section{Case Report}

A 65-year-old male presented with complaints of severe headache for 10 days. Past history revealed a minor fall

\begin{tabular}{|l|l|}
\hline \multicolumn{2}{|c|}{ Access this article online } \\
\hline Quick Response Code: & Website: \\
\hline & www.ruralneuropractice.com \\
\cline { 2 - 3 } & \\
\hline
\end{tabular}

with head injury 1 month ago for which no medical attention was sought. Examination was unremarkable, except for the presence of pronator drift on the left side. CT (computed tomography) of the head showed a right cSDH, measuring $15 \mathrm{~mm}$ in thickness and causing a $7 \mathrm{~mm}$ midline shift to the left [Figure $1 \mathrm{a}$ and $\mathrm{b}$ ]. After baseline investigations which documented a normal coagulation profile, the patient was taken for right frontal and parietal burr holes and drainage of the subdural hematoma under MAC (monitored anesthesia care) with conscious sedation. After making the burr holes, the dura was coagulated with bipolar cautery and incised in a cruciate manner. The cut edges of the dura were further cauterized to shrink the dura and keep the dural opening patent. The subdural collection was allowed to drain without any negative pressure being applied in the subdural cavity. After the subdural hematoma had drained on its own accord (owing to the pressure differential), the cavity was gently irrigated with normal saline till the returning fluid was clear. The surgery was uneventful and hemostasis was achieved prior to wound closure. The patient's hemodynamic parameters were stable throughout the procedure. CT head done immediately post-surgery revealed a subependymal bleed in the right lateral ventricle, extending laterally into the thalamus and medially into the body of the lateral ventricle [Figure $1 \mathrm{c}$ and $\mathrm{d}$ ]. In spite of these findings in the post-operative $\mathrm{CT}$, the patient did well in the

Address for correspondence:

Dr. Amey R. Savardekar, Department of Neurosurgery, Post Graduate Institute of Medical Education and Research, Chandigarh, Punjab, India. E-mail: ameysavardekar@gmail.com 


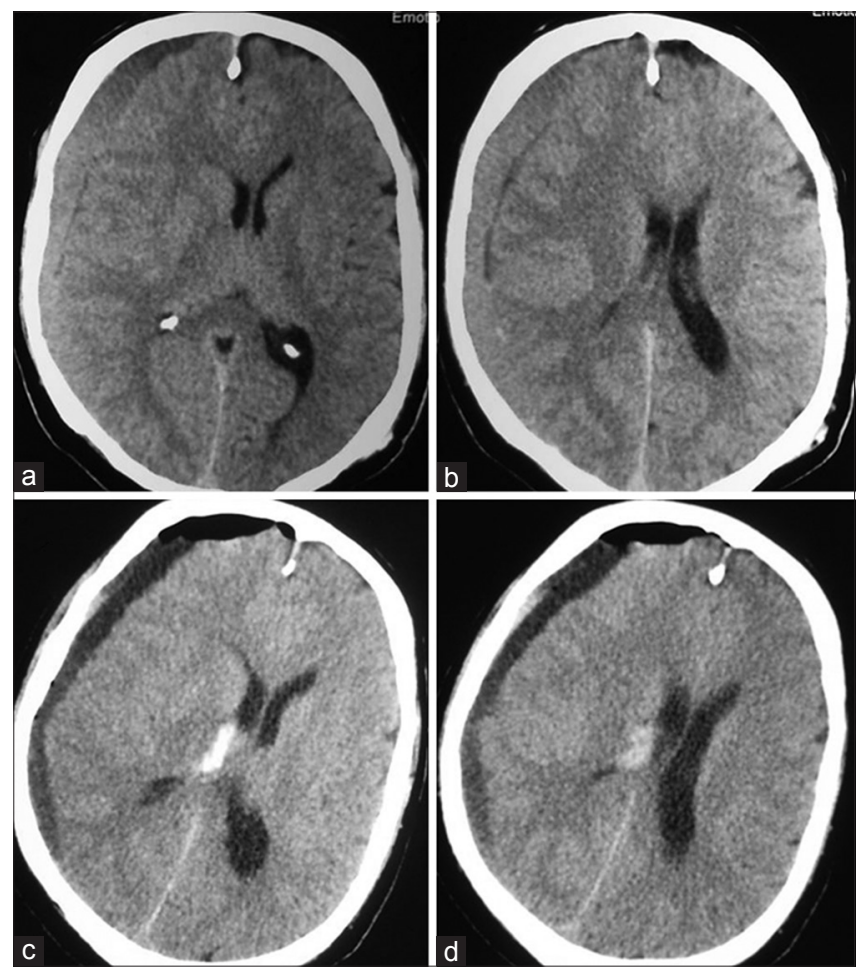

Figure 1: $(a$ and $b)$ Pre-operative CT head showing right chronic subdural hematoma. (c and d) Post-operative CT head showing subependymal bleed in the right thalamus with extension into the lateral ventricle

post-operative period with complete resolution of headache and improvement of the subtle pyramidal deficit on the left side.

\section{Discussion}

The prognosis of cSDH is relatively good. ${ }^{[1]}$ Some complications, as mentioned above, do tend to jeopardize the good outcome normally expected in these patients; but none are as devastating as the rare complication of intracerebral hemorrhage (ICH) after evacuation of $\mathrm{cSDH} .{ }^{[2,5]}$ Several causative mechanisms have been postulated to explain this complication following a simple surgical procedure. Beneath the cSDH, focal cerebral edema due to impeded venous drainage can reduce cerebral blood flow in the ipsilateral hemisphere. Chronic dilatation of small arterial vessels and loss of carbon dioxide reactivity in the ischemic hemisphere could also contribute to the pathogenesis. ${ }^{[6]}$ Possible pathogenic mechanisms, for $\mathrm{ICH}$ in the thus compromised brain, include hemorrhage into previously undetected areas of contusion, damage to cerebral vasculature secondary to rapid peri-operative parenchymal shift, and sudden increase in cerebral blood flow (post-decompression) combined with focal disruption of autoregulation. ${ }^{[2,3,7]}$
Compression by extra-axial collection decreases cerebral blood flow on the affected hemisphere and alters its vascular self-adjustment. ${ }^{[6,7]}$ Some authors hypothesize that the rapid increase in cerebral blood flow (after drainage) in areas of the brain with altered vascular self-adjustment may be the most likely precipitating mechanism of intracerebral hemorrhage after surgical evacuation of cSDHs. ${ }^{[2,7]}$ This mechanism could have resulted in the subependymal bleed in our patient. However, as the compressed brain is more toward the surface, this mechanism is likely to explain superficial or lobar hematomas. Our case exhibited a subependymal bleed, post-drainage. We hypothesize that after sudden decompression of the brain, the differential expansile qualities of the solid (brain) and liquid (CSF) components of the cranium may result in mechanical stress at the interface, which in turn, may cause rupture of the engorged subependymal veins (due to prolonged compression). This may explain the post-operative CT finding in our case.

Chronic SDH being a benign entity, neurosurgical outcome have to be optimized for this entity. Considering that intracerebral hemorrhage inadvertently portends a poor outcome for the affected patients, preventive steps are imperative. ${ }^{[2,3]}$ These include gradual and graded decompression of the chronic SDH (especially for large collections), use of closed drainage system which facilitates slow re-expansion of the brain, and careful control of blood pressure lability in the peri-operative period. The gradual and graded decompression can be achieved by covering the dural aperture with a cottonoid after draining around 15 to $20 \mathrm{~mL}$ of the subdural hematoma. After waiting for approximately $5 \mathrm{~min}$, the cottonoid can be removed to allow further drainage of 15 to $20 \mathrm{~mL}$. This procedure can be continued till the subdural pressures equalize with the atmospheric pressures, and at this juncture, saline washes can be instituted. This especially holds true for very large subdural hematomas, presenting with raised intra-cranial pressure. Our patient was fortunate to have a good outcome as the bleed was moderate in size, however, we advocate taking steps to prevent such complications altogether.

\section{References}

1. Mori K, Maeda M. Surgical treatment of chronic subdural hematoma in 500 consecutive cases: Clinical characteristics, surgical outcome, complications, and recurrence rate. Neurol Med Chir (Tokyo) 2001;41:371-81.

2. d'Avella D, De Blasi F, Rotilio A, Pensabene V, Pandolfo N. Intracerebral 
hematoma following evacuation of chronic subdural hematomas. Report of two cases. J Neurosurg 1986;65:710-2.

3. Muneza S, Rasoloherimampiononiaina MR, Nduwamariya MJ. Postoperative intracerebral and intraventricular hemorrhages following removal of a chronic subdural hematoma. J Clin Neurosci 2009;16:1346-8.

4. Ulivieri S, Oliveri G. Intracerebral haemorrhage following surgical evacuation of chronic subdural haematoma: Case report. G Chir 2008;29:233-4.

5. Sousa J, Golash A, Vaz J, Chaudhary H. Spontaneous intracerebral haemorrhage following evacuation of chronic subdural hematomas. J Clin Neurosci 2004;11:794-6.

6. Ishikawa T, Kawamura S, Hadeishi H, Suzuki A, Yasui N, Uemura K.
Cerebral blood flow and oxygen metabolism in hemiparetic patients with chronic subdural hematoma. Quantitative evaluation using positron emission tomography. Surg Neurol 1995;43:130-7.

7. Kim JK, Kim SW, Kim SH. Intracerebral hemorrhage following evacuation of a chronic subdural hematoma. J Korean Neurosurg Soc 2013;53:108-11.

How to cite this article: Savardekar AR, Salunke P. Subependymal hemorrhage following drainage of chronic subdural hematoma: Probable causative mechanisms and prevention strategies. J Neurosci Rural Pract 2015;6:252-4.

Source of Support: Nil. Conflict of Interest: None declared. 